\section{Applicability of the dielectric barrier discharge for helium ash measurements in the divertor region}

Ireneusz Książek, Andrzej Brosławski, Henryk Janus, Ewa Pawelec

\begin{abstract}
Controlled fusion based on the magnetic confinement of the plasma is one of the main aims of the Eurofusion programme. In the fusion device, the hydrogen isotopes, in nuclear reactions, will produce helium nuclei. The products, as the ash, will be removed from the plasma in the region of the so-called divertor. Controlling the helium to hydrogen ratio in this 'exhaust gas' will provide information about the efficiency of the fusion process as well as of the efficiency of the helium removal system. One of the methods to perform this task is to study the properties of the discharge conducted in such exhaust gas. In this paper, the applicability of the dielectric barrier discharge (DBD) is studied. This preliminary experiment shows a great potential in applicability of this kind of discharge. The optical as well as pulse-height spectra were studied, both revealing very promising properties. In the optical spectrum, one can observe well separated hydrogen and helium spectral lines, with intensities of the same order of magnitude. Moreover, in the registered spectral region, the molecular spectra are negligible. The pulse-height spectra reveal very distinct shape in helium and hydrogen. Checking of this spectrum could provide parallel (redundant) information about the partial pressure of helium in the magnetic confinement fusion (MCF) device exhaust gas.
\end{abstract}

Key words: dielectric barrier discharge (DBD) $\bullet$ low pressure discharge $\bullet$ fusion plasma

I. Książek ${ }^{\bowtie}$, A. Brosławski, H. Janus, E. Pawelec Institute of Physics,

Opole University,

48 Oleska Str., 45-052 Opole, Poland,

Tel.: +48 77452 7263, Fax: +48 77452 7290,

E-mail: iksiaz@uni.opole.pl

Received: 25 September 2015

Accepted: 17 November 2015

\section{Introduction}

One of the important issues of operating fusion power plants is to control the composition of the fusion plasma [1]. The fusion processes are associated with creation of helium nuclei (alpha particles) in nuclear reactions of hydrogen isotopes (deuterium-tritium as well as deuterium-deuterium). In the reaction considered, that is, deuterium-tritium, hot alpha particles produced in these reactions dissipate heat to the plasma, which is a process needed for plasma sustaining, but afterwards they ought to be removed to prevent fuel dilution. It could be achieved by appropriate configuration of the magnetic field pushing out the helium atoms associated with the exhaust system providing effective removal of the plasma fusion 'ash', i.e. alpha particles. The concentration of the helium in the fusion plasma (plasma core) cannot exceed $10 \%$ [1].

The amount of the helium inside the plasma vessel can be measured, e.g. by emission or charge exchange spectroscopy. Simultaneous monitoring of the gas composition in exhaust system would provide additional information about the efficiency of the ash removing system, including magnetic field configuration, and can be applied in a feedback loop preventing critical decrease of the efficiency of the fusion plasma reactor. 
One of the most precise methods of the gas composition analysis is mass spectroscopy. Unfortunately, the ratio of charge to mass in the case of helium atoms is very close to the value of this parameter for the deuterium molecule, so they cannot be easily distinguished by this technique. The more sophisticated mass spectroscopy methods have very low time resolution (order of minutes [1]) and their operation could be easily disturbed by magnetic fields present in the vicinity of the magnetic plasma confinement device.

An alternative method could be the spectral analysis of the radiation emitted by exhaust gas. In order to register such radiation, one needs to apply a proper excitation source. The study of different discharge sources was performed by Finken and Denner [1,2] with the conclusion that the best is the Penning discharge associated with the operation of the Penning-type gauge. Unfortunately, this method has some serious disadvantages. It could be applied only for very low pressures, due to conditions required by Penning discharge. Another difficulty that is observed in this discharge is the overlapping of the helium line and the hydrogen molecular spectrum [2].

The barrier discharge is commonly used in plasma chemistry, both in industry and as laboratory chemical reactors. One of its main advantages is a simple scalability from small laboratory devices into large industrial installation. In most cases, the discharge is conducted under the atmospheric pressure [3]. Another common application is the surface treatment (e.g. $[4,5])$ or sterilisation of the surfaces in medicine (e.g. [6, 7]). This type of discharge was also applied as an excitation source in study of the atomic spectra and determination of the atomic constants, especially for the rare earth elements, e.g. $[8,9]$.

The main aim of this paper is to study the possibility of applying the barrier discharge, with the barrier containing the ferroelectric insulator, as the excitation source of helium and hydrogen atoms. The advantage of this source is the discharge chamber can be constructed with almost unlimited variety of shape as well as geometry of electrodes (cylindrical, flat-flat, flat-spike, etc.). Moreover the costs of the device are relatively low.

The experiment was performed applying a range of gas pressures as well as the helium to hydrogen ratio in the gas mixture. For the preliminary experiment, we decided to use the hydrogen instead of deuterium. The deuterium, as a hydrogen isotope, has the same chemical properties (and very similar spectral properties) as deuterium and hydrogen is much more abundant and available. Simultaneous to the spectroscopic measurements, the pulse height spectra of the discharge pulses were studied.

\section{Experimental set-up}

The general scheme of the experimental set-up is presented in Fig. 1. The discharge was conducted between two flat electrodes made of the $\mathrm{BaTiO}_{3}$ ce-

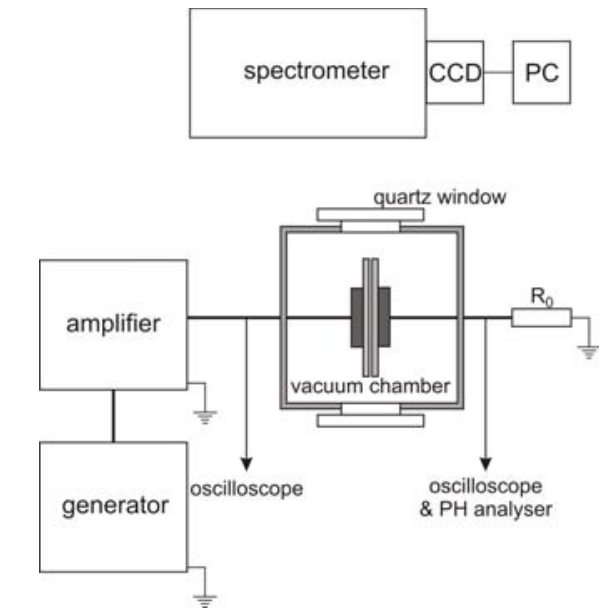

Fig. 1. Diagram of the experimental set-up.

ramics (thickness $1 \mathrm{~mm}$, dielectric constant 1300). The distance between the electrodes was $0.5 \mathrm{~mm}$. The photo of the chamber is presented in Fig. 2.

The input signal was sinusoidal with the frequency of $500 \mathrm{~Hz}$. The input voltage was adjusted manually by setting the lowest voltage to conduct the stable discharge. One of the technical difficulties was the appearance of parasitic discharges between the electrodes and the chamber construction elements. This phenomenon restricted the lowest pressure for which the stable operation of the device was possible. This drawback may be overcome by modifying the mechanical construction of the chamber.

The initial gas inlet system allows filling up the chamber with pure helium, pure hydrogen or initially prepared mixture of helium and hydrogen (1:1 by volume). After modification of the system, it was possible also to change the gas mixture composition by admixing to the $\mathrm{He}+\mathrm{H}_{2}$ mixture either pure helium or pure hydrogen. By damping the output by a flow resistor (needle valve), one could adjust the pressure inside the chamber. Similar system, allowing slight increase of the pressure in the discharge chamber, could be also applied for analysing of the exhaust provided by the divertor or limiter pumps.

The optical spectra were recorded using a grating spectrometer (PGS-2) equipped with a CCD detector. The time of the registration was $30 \mathrm{~s}$. The dispersion of the system allows to register simultaneously a spectral range of approximately $35 \mathrm{~nm}$. In the preliminary experiment, several ranges of the spectra

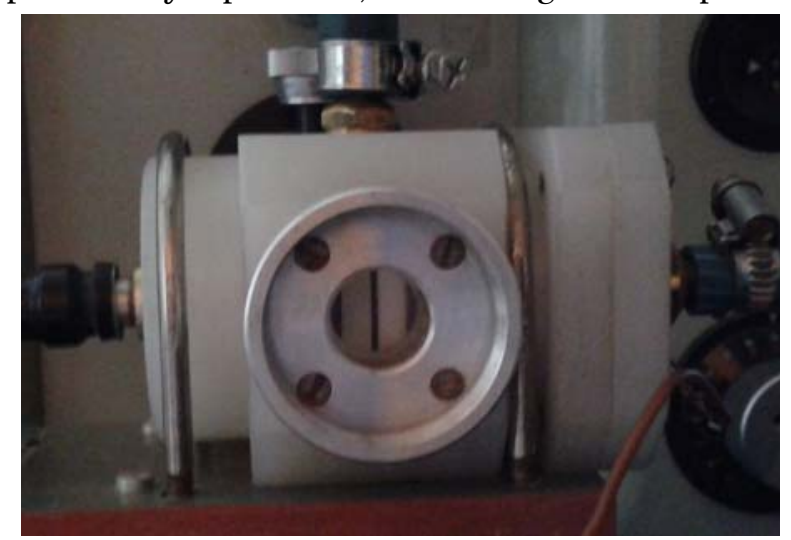

Fig. 2. External view of the discharge chamber. 


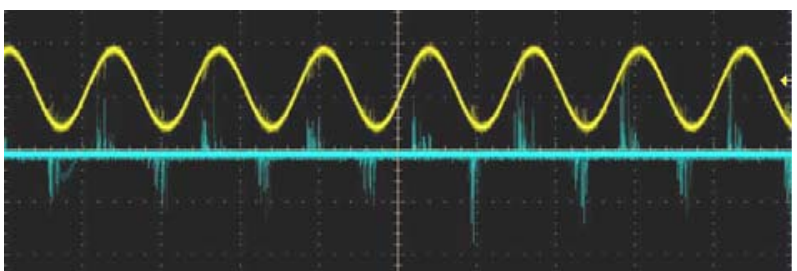

Fig. 3. Sample of the oscillogram. The sinusoidal (upper, yellow) waveform represents the input voltage; the line with spikes (lower, blue) represents voltage changes on the output resistor associated with the discharge current.

were registered. Based on these preliminary results, it was decided to concentrate on a spectral region with the $\mathrm{H}_{\alpha} 656.3 \mathrm{~nm}$ and $\mathrm{HeI} 667.8 \mathrm{~nm}$.

The input voltage signal was registered by the first channel of the digital oscilloscope. The second channel was used for the measurements of the voltage drop on the $100 \Omega$ output resistor (which is proportional to the discharge current). This signal was simultaneously fed to the pulse-height analyser (AMPTEC DP5), which allows record and study the pulse-height spectra of the discharge. The sample oscillogram in Fig. 3 presents the sinusoidal input and the current pulses associated with the microdischarges between the electrodes.

\section{Results}

The sample spectrum is presented in the Fig. 4. One needs point out that in a very wide range of the pressures as well as the gas compositions (i.e. an amount of helium in the mixture), the intensities of both selected lines are of the same order of magnitude. One of the problems described in the Denner paper [2] was a very large difference between intensities of hydrogen and helium line (more than one order of magnitude) registered in the Penning discharge. The registered spectra show also that the hydrogen molecular spectra are negligibly small (again unlike in the case of Penning source). The lines are very well separated, which allows to application of a simple system (with low spectral resolution) in order to measure the ratio of the line intensities. Before one puts such a system into operation, one needs to check carefully the spectrum for presence of spectral lines of fusion plasma impurities.

In Figs. 5 and 6 , one can observe that the dependence of the intensities of the registered lines on helium's partial pressure is different for different total pressures in the discharge chamber. It can be associated with two modes (or types) of barrier dis-

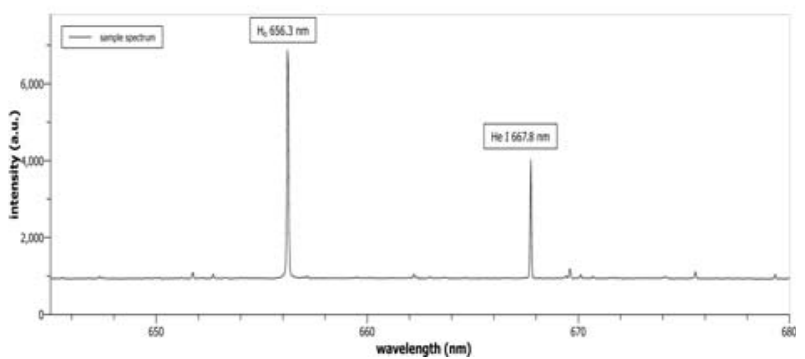

Fig. 4. Sample of the registered spectrum.

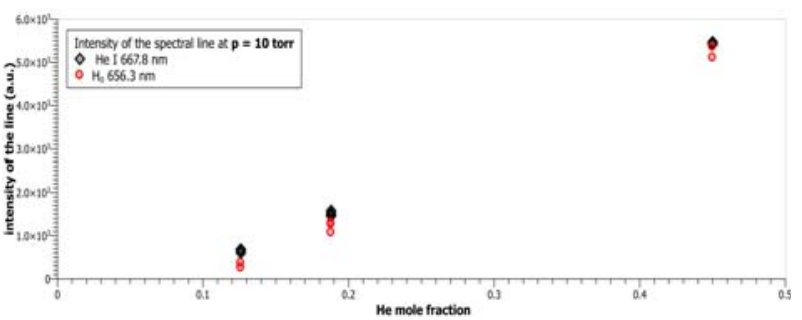

Fig. 5. Dependence of the registered lines intensities on the amount of $\mathrm{He}$ in the gas mixture at pressure in the discharge chamber of 10 torr.

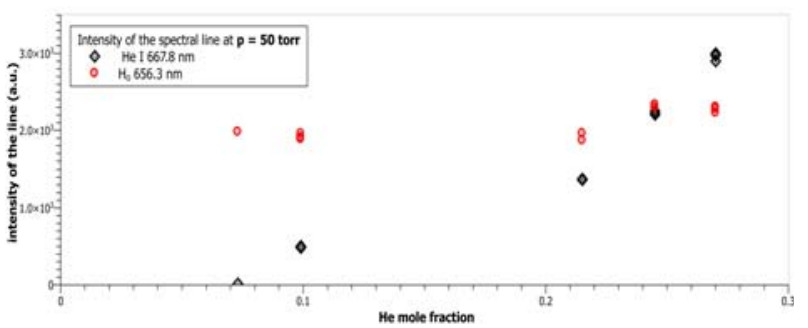

Fig. 6. Dependence of the registered lines intensities on the amount of $\mathrm{He}$ in the gas mixture at pressure in the discharge chamber of 50 torr.

charge called 'glow discharge DBDs' and 'Townsend-like DBDs' [10]. Unfortunately, both cases seem to reveal non-linear dependence, so determination of the helium amount based on spectroscopic measurement will require careful calibration. For pressures above 20 torr, the line intensities are fairly constant, as presented in Fig. 7 .

The radius of the single micro-discharge is proportional to product of the reciprocal value of the product of the gas density and the derivative of the effective ionisation coefficient [3]. The parameters of the micro-discharge depend on properties of the dielectric as well as on the pressure and composition of the gas mixture in the chamber. This dependency reflects in the pulse-height spectrum of the micro-discharges. There are significant differences between physical properties of helium (as monoatomic gas with very high first ionisation potential) and hydrogen (diatomic molecules with much lower first ionisation potential). It can explain why the pulse-height spectra registered in a pure helium and hydrogen are significantly different (see Fig. 8).

In a case of a small admixture of helium to hydrogen, one can observe a shift of the maximum of the pulse-height depending on the amount of helium (see Fig. 9).

The presented pulse-height spectra were obtained by integration (counting) data over the period of

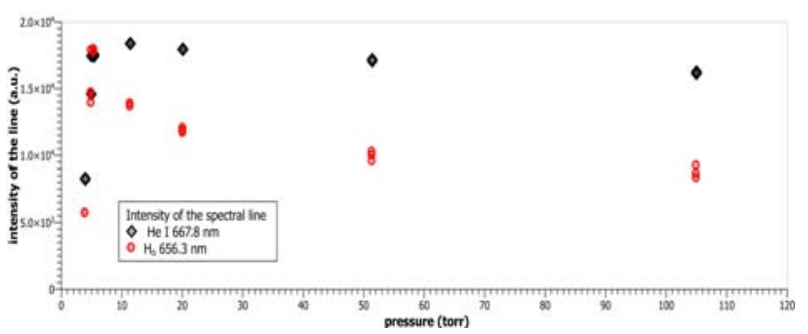

Fig. 7. Dependence of the lines intensities registered for mixture $\mathrm{He}+\mathrm{H}_{2}(1: 1$ by volume) on the pressure in the discharge chamber. 


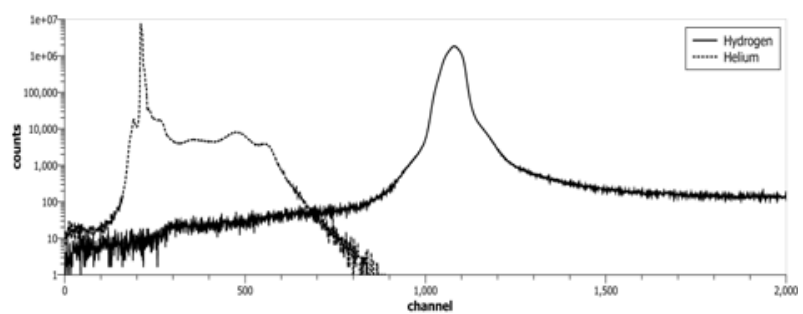

Fig. 8. Comparison of the pulse-height spectra registered in pure helium (dashed line) and pure hydrogen (solid line) at the pressure in the discharge chamber of 10 torr.

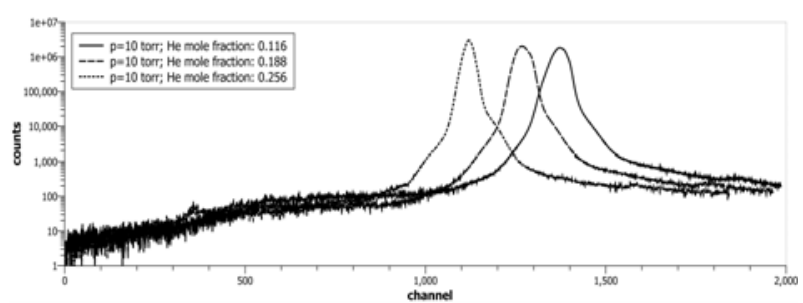

Fig. 9. Pulse-height spectra registered in different mixtures of helium and hydrogen at the pressure in the discharge chamber of 10 torr.

$120 \mathrm{~s}$. The analysis of time dependences of the pulse-height data may also be considered.

\section{Conclusions}

These preliminary studies show a potential applicability of the barrier discharge for determining the composition of the hydrogen-helium mixture, as e.g. in exhaust line of the plasma fusion machine. The results obtained by optical spectroscopy reveal a non-linear dependency of the measured lines intensities as function of the pressure as well as the helium amount in the gas mixture. This inconvenience can be easily overcome by calibration of the future measurement device.

The results of the pulse-height spectra measurements are very promising. The spectra registered in the pure hydrogen differ significantly from those obtained in pure helium. Small admixture of helium in hydrogen results in a distinct shift of the maximum of the pulse-height spectrum.

The detailed theoretical description of the spectral as well as electrical properties of such discharge is very complicated. The plasma is very far from thermal equilibrium and moreover the low pressure of the discharge and complex interactions between plasma constituents (molecular gas - hydrogen and atomic helium) results in a very complex model. Moreover, the quantitative as well as qualitative description of the discharge strongly depends on the pressure. This very interesting task is beyond the scope of this work and, if this research is decided to be continued, the theoretical description of the processes will also be developed.

Acknowledgments. This work has been carried out within the framework of the EUROfusion Consortium and has received funding from the Euratom research and training programme 2014-2018 under grant agreement no. 633053. The views and opinions expressed herein do not necessarily reflect those of the European Commission. This scientific work was partly supported by the Polish Ministry of Science and Higher Education within the framework of the scientific financial resources in the year 2014 allocated for the realization of the international co-financed project.

This work was performed at the Opole University, Poland.

\section{References}

1. Finken, K. H., Dippel, K. H., Baek, W. Y., \& Hardtke, A. (1992). Measurement of helium gas in a deuterium environment. Rev. Sci. Instrum., 63(1), 1-7.

2. Denner, T., Finken, K. H., \& Mank, G. (1996). Helium partial pressure measurement in a deuterium environment. Rev. Sci. Instrum., 67(10), 3515-3520.

3. Kogelschatz, U. (2003). Dielectric-barrier discharges: Their history, discharge physics and industrial applications. Plasma Chem. Plasma Process., 23 (1), 1-46.

4. De Geyter, N., Morent, R., \& Leys, C. (2006). Surface modification of a polyester non-woven with a dielectric barrier discharge in air at medium pressure. Surf. Coat. Technol., 201, 2460-2466.

5. Morent, R., De Geyter, N., Leys, C., Gengembre, L., \& Payen, E. (2007). Surface modification of non-woven textiles using a dielectric barrier discharge operating in air, helium and argon at medium pressure. Text. Res. J., 77(7), 471-488.

6. Fridman, G., Peddinghaus, M., \& Ayan, H. (2006). Blood coagulation and living tissue sterilization by floating-electrode dielectric barrier discharge in air. Plasma Chem. Plasma Process., 26, 425-442.

7. Eto, H., Ono, Y., \& Ogino, A. (2008). Low-temperature internal sterilization of medical plastic tubes using a linear dielectric barrier discharge. Plasma Process. Polym., 5(3), 269-274.

8. Kusz, J. (1992). Intensities and transition probabilities for selected DyI and DyII lines emitted from a ferroelectric plasma source. Astron. Astrophys. Suppl. Ser., 92, 517-532.

9. Goly, A., Kusz, J., Nguyen Quang, B., \& Weniger, S. (1991). Transition probabilities of PrII-lines emitted from a ferroelectric plasma source. J. Quant. Spectrosc. Radiat. Transfer, 45(3), 157-163.

10. Kogelschatz, U. (2010). Collective phenomena in volume and surface barrier discharges. J. Phys. Conf. Ser., 257, 1-12. DOI: 10.1088/1742-6596/257/1/012015. 